\title{
Papouasie Nouvelle-Guinée / Australie : des relations ambivalentes
}

\section{Rémy Herrera et Poeura Tetoe}

\section{Q OpenEdition \\ 1 Journals}

Édition électronique

URL : http://journals.openedition.org/jso/6689

DOI : $10.4000 /$ jso.6689

ISSN : $1760-7256$

Éditeur

Société des océanistes

\section{Édition imprimée}

Date de publication : 31 décembre 2012

Pagination : 201-214

ISBN : 978-2-85430-033-8

ISSN : 0300-953x

\section{Référence électronique}

Rémy Herrera et Poeura Tetoe, "Papouasie Nouvelle-Guinée / Australie : des relations ambivalentes », Journal de la Société des Océanistes [En ligne], 135 | 2012-2, mis en ligne le 18 février 2013, consulté le 19 avril 2019. URL : http://journals.openedition.org/jso/6689 ; DOI : 10.4000/jso.6689

(c) Tous droits réservés 


\title{
Papouasie Nouvelle-Guinée / Australie : des relations ambivalentes
}

\author{
par
}

\author{
Rémy HERRERA* et Poeura TETOE**
}

\section{RÉSUMÉ}

Le présent article interroge l'ambivalence des relations entre la Papouasie Nouvelle-Guinée et l'Australie, en analysant successivement les liens historiques inégaux qui les attachent ( $1^{\text {ère }}$ partie), leur continuité par delà l'indépendance ( $2^{e}$ partie) et les mécanismes de cette dépendance, spécialement sous ses aspects économico-politiques ( $3^{e}$ partie). Les structures sociales de l'ancienne colonie australienne sont analysées notamment en liaison avec les questions de l'accès à la terre et de l'essor du secteur minier pénétré par le capital étranger, autour desquels s'articulent et s'entrechoquent les intérêts des États et des transnationales, d'une part, et ceux de la population papouane néo-guinéenne, d'autre part.

Mots-CLÉS : développement, dépendance, ressources naturelles, Papouasie Nouvelle-Guinée, Australie

L'Australie est une puissance régionale importante, dont la sphère d'influence directe couvre pour l'essentiel la Papouasie NouvelleGuinée, le sud-ouest du Pacifique Sud (Salomon, Vanuatu, Fidji, Tuvalu, Nauru, Kiribati, en plus de la mer de Corail, Norfolk et Lord Howe, et de quelques dépendances dans l'océan Indien) et, en Asie du Sud-Est, le Timor oriental. Mais ses intérêts de défense nationale officiellement reconnus s'étendent sur un large triangle Pakistan / Inde, Sibérie russe / Chine et Pacifique - y compris l'Indonésie (Herrera, 2007). L'acceptation de cette zone par ses alliés n'exclut pas certaines rivalités et tensions ponctuelles

\section{ABSTRACT}

This article studies the ambivalency of the relationships between Papua New Guinea and Australia, by analyzing successively their inequal historical links $\left(1^{\text {st }}\right.$ part $)$, the continuity of the latter after the independency ( $2^{\text {nd }}$ part), and the mechanisms of the dependency, especially at their economic and political levels $\left(3^{\text {rd }}\right.$ part). The social structures of the former Australian colony are analyzed, in particular in relation to the questions of the access to land and to the expansion of the mining sector penetrated by the foreign capital, around which the interests of the states and the transnational firms, on the one hand, and those of the PNG people, on the other hand, are clashing.

Keywords: development, dependency, natural resources, Papua New Guinea, Australia
- notamment avec la France, qui garde des possessions dans cette région, ou la GrandeBretagne, toujours influente sur ses anciennes colonies. Cette mission de «stabilisation régionale» est assurée en partenariat avec la Nouvelle-Zélande, dont les intérêts immédiats se situent plutôt en Polynésie (Samoa, Tonga, îles Cook, Niue, Tokelau).

Les États-Unis enserrent l'ensemble par un gigantesque arc de cercle, au-delà de Hawaii, des atolls Johnston, Wake et Midway sous administration militaire et des Line Islands, grâce au contrôle qu'ils exercent sur Palau, Guam, la fédération de Micronésie, le Commonwealth des

* Chercheur au CNRS, umR 8174 Centre d'Économie de la Sorbonne, herrera1@univ-paris1.fr

** Doctorante en Sciences économiques, UMR 8174 Centre d'Économie de la Sorbonne, mikimiki@lagoon.nc 
Mariannes du Nord et les Samoa américaines. En outre, l'Australie, entretenant des relations privilégiées avec Washington, accueille officiellement au moins une base militaire états-unienne, située à Darwin, ainsi qu'un centre d'écoute à Pine Gap, près d'Alice Springs. Des sous-marins états-uniens mouillent à Sterling Bay ou à Darwin, et des exercices militaires conjoints sont régulièrement réalisés avec l'Australian Defense Force dans la mer de Corail et différents camps militaires du Queensland (Shoalwater Bay, Townsville Field Training Area...). L'interopérationalité est devenue le maître-mot de la restructuration de l'armée australienne, de plus en plus intégrée au dispositif militaire global des États-Unis. Dans cette optique, le système de défense australien s'est récemment renforcé et modernisé (dépenses et effectifs accrus, matériels sophistiqués, formations actualisées...) et la stratégie militaire a été modifiée (la forward defence de la guerre froide faisant place à la pro-active defence), pour faire face à tout risque d'instabilité politique régionale (Australia Government, 2009).

$\mathrm{Au}$ plan économique, depuis plus de deux décennies, les capitaux provenant d'Australie se dirigent davantage vers l'Asie émergente, en restant encore très importants aux États-Unis, au Canada, en Grande-Bretagne et en NouvelleZélande (Economist Intelligence Unit, années variées). Ceux investis dans le Pacifique en développement demeurent toutefois rentables, tout spécialement en Mélanésie, qui a représenté historiquement la base de l'expansion du capitalisme australien en direction de l'Asie et reste un objectif fondamental pour Canberra. Cette chasse gardée constitue un débouché assuré pour les exportations de l'Australie, qui domine les secteurs clés de plusieurs pays de la région, notamment dans les mines. Pourtant, ce phénomène est sans doute le plus visible en Papouasie Nouvelle-Guinée ou Papua Niugini en pidgin (6,5 millions d'habitants pour $463000 \mathrm{~km}^{2}$ ), sur laquelle nous nous concentrerons dans le présent article. Nous montrerons l'ambivalence des relations entre ce pays et l'Australie, en analysant successivement les liens qui les attachent (première partie), leur continuité après l'indépendance (deuxième partie) et les mécanismes de cette dépendance (troisième partie).

\section{Les liens de l'histoire}

Ce n'est que dans la seconde moitié du $\mathrm{XIX}^{\mathrm{e}}$ siècle que l'Australie coloniale, bénéficiant de flux massifs de capitaux et de migrants venus du
Royaume-Uni, commença à s'intéresser aux richesses qui faisaient alors la réputation de la Nouvelle-Guinée : bois, gommes, perles, et surtout coprah. Dès la fin des années 1860 avaient été fondées à Sydney plusieurs compagnies commerciales spécialisées dans le négoce avec l'île du Nord. L'Europe avait « découvert» la NouvelleGuinée trois siècles et demi auparavant, par ses navigateurs portugais (Abreu en 1511 et Meneses en 1527, lequel crut toucher la «terre australe » des récits de Marco Polo), puis espagnols (avec Ortiz de Retes, qui donna à l'endroit son nom moderne - les autochtones lui rappelant ceux des côtes africaines du golfe de Guinée - et l'annexa en 1546 au royaume d'Espagne). Ces premiers contacts eurent toutefois lieu sans colonisation de peuplement, ni occupation militaire. À partir du milieu du XVII siècle, à la suite des passages de Le Maire, Schouten et Tasman, l'île allait être revendiquée par les jeunes Provinces-Unies, qui venaient de gagner leur indépendance vis-à-vis de l'Espagne après l'Unie van Utrecht de janvier 1579. Les voyages ultérieurs de Dampier, lors des missions d'exploration de l'Australie en 1688-99, puis de Cook, qui effectua la circumnavigation de la Nouvelle-Guinée en 1770 (soit l'année de la découverte de la péninsule de Banks en Nouvelle-Zélande) marquèrent l'intérêt porté par l'Angleterre à cette terre lointaine et conduisirent à son annexion à la fin du XVIII ${ }^{\mathrm{e}}$ siècle lorsque la British East India Company s'y implanta. La colonisation effective ne débuta cependant qu'en 1828 avec l'occupation de la partie Nord-Ouest par les Pays-Bas, qui, à partir de leur colonie des Indes orientales (NederlandsIndië) avaient évincé les sultans malais des Moluques. Les flux de colons européens, à compter du milieu du $\mathrm{XIX}^{\mathrm{e}}$ siècle, provenaient surtout de Grande-Bretagne au sud-est, dans la région de Port Moresby, et d'Allemagne sur la côte septentrionale.

Les liens qui allaient attacher la Papouasie Nouvelle-Guinée à l'Australie se resserrèrent à la fin $\mathrm{du} \mathrm{XIX}^{\mathrm{e}}$ siècle. Enchaînant sur les cycles aurifères australiens - qui donnèrent jusqu'à $40 \%$ des minerais extraits dans le monde à partir de 1850-60 (INSEE, 1951) -, une première ruée vers l'or déferla sur la Nouvelle-Guinée en 1877. Mais le moteur qui propulsa de façon décisive la colonisation de la Nouvelle-Guinée par l'Australie fut la pression exercée par la rivalité des autres puissances coloniales. Les sociétés allemandes s'activaient sur les côtes Nord, et la France était présente dans la région, notamment en Nouvelle-Calédonie (Leblic, 1993). Inquiets, les colons du Queensland s'employèrent à 
convaincre l'Angleterre, en vain dans un premier temps, d'occuper la partie orientale. En 1883, quelques mois seulement avant la conférence de Berlin, sans attendre l'aval ni d'ailleurs obtenir le soutien de la métropole, ils annexaient ces territoires. Ce ne fut qu'à la suite de l'affirmation grandissante des intérêts allemands - notamment commerciaux - dans le Pacifique que Londres prit officiellement possession de la région sud-orientale (Pons, 1994). L'Allemagne riposta immédiatement par l'annexion de la partie nord-orientale de la Nouvelle-Guinée (protectorat de Kaiser-Wilhelmsland), ainsi que des archipels Bismarck et des Salomon en 1884 et des îles Marshall en 1885. En 1886, une convention anglo-allemande était signée à Berlin, qui fixait les sphères d'influence respectives entre Britanniques et Allemands; et, en 1886, étaient négociées et tracées les frontières séparant Nouvelle-Guinée hollandaise à l'ouest, territoires allemands au nord-est et Papouasie anglaise au sud-est. Cette dernière portion, étendue le long du $141^{\mathrm{e}}$ degré de longitude Est au profit des Britanniques, fut remise par la Grande-Bretagne à l'Australie en 1905.

Avant cela, au $\mathrm{XIX}^{\mathrm{e}}$ siècle, comme en tant d'autres endroits de la Mélanésie, les populations indigènes avaient subi le blackbirding (Molesworth, 1917 ; Panoff, 1979 ; Maude, 1981), soit ce système de recrutement forcé de maind'œuvre destinée aux plantations coloniales ou aux cannaies sucrières du Queensland. Les villages des côtes et des îles avoisinantes furent ainsi saignés de dizaines de milliers d'hommes, réduits au statut d'indenture - assez proche de l'esclavage. Chaque puissance coloniale se réservait une zone de contrôle de l'accès à la maind'œuvre servile par les navires recruteurs: l'Allemagne, l'archipel Bismarck, les Salomon, Bougainville, Buka ; l'Angleterre, la Papouasie elle-même. Plus tard, face aux résistances locales (Gille et Toulellan, 1999), les besoins de maind'œuvre conduisirent les planteurs à déplacer des travailleurs asiatiques, sous contrats de salariat bridé, cette fois en provenance de Chine, d'Inde ou de Malaisie, entre autres. En Papouasie et Nouvelle-Guinée, c'est surtout la culture du coprah, plus que celles de café, de cacao et de caoutchouc, qui était développée, pour l'exportation vers les métropoles de tutelle, via des comptoirs et des compagnies privées, par exemple, la société à charte berlinoise Deutsche Neuguinea Kompagnie de 1884 à 1914. Les plantations s'étendirent dans la région de Madang (anciennement Friedrich-Wilhelmshafen) et sur les archipels environnants (Neu-Pommern, NeuMecklenburg, Salomon...). Avec les découvertes aurifères et l'abondance de terres, cet approvisionnement en travailleurs forcés étrangers se révéla tout à fait décisif pour le développement de l'Australie et son passage - non bloqué par sa métropole au XIX ${ }^{\mathrm{e}}$ siècle - d'une économie exportatrice de biens primaires à une structure aux industries diversifiées, puis tertiarisée. Après 1904-06 et l'ostracisme du droit du travail australien, la fermeture de l'Australie à la maind'œuvre non blanche par la White Policy, illustration de la discrimination raciale de l'époque, inversa les flux de travailleurs : nombre de Mélanésiens embauchés jadis dans les sucreries du Queensland furent expulsés - même s'il reste aujourd'hui des descendants de ces islanders, distincts des Aborigènes, dans le Nord. Dans le même temps, cette politique s'adapta aux intérêts commerciaux australiens dans la région, en interdisant l'entrée aux concurrents potentiels venus d'Asie.

Les expéditions minières se multiplièrent en British New Guinea. La première découverte importante d'or, par un résident du North Queensland, eut lieu sur l'île de Sudest, en 1888. Puis, au tournant $\mathrm{du} \mathrm{xx}^{\mathrm{e}}$ siècle, les routes des aventuriers se déplacèrent vers la grande île, gagnant peu à peu l'intérieur des terres vers l'Ouest, provoquant un nouveau rush - au prix de durs conflits avec les populations locales. Dès l'éclatement de la Première Guerre mondiale, l'armée australienne occupa les territoires allemands de Nouvelle-Guinée et de Bismarck, avant que la Société des Nations lui en remît le mandat en 1921. Les commandes militaires et l'isolement relatif du pays avaient contribué à concentrer fortement le capital, et l'Australie se constitua un " empire », relayant l'Angleterre et partant à la conquête économique de ses colonies du Pacifique. En Papouasie et NouvelleGuinée, les incitations financières accordées par l'administration coloniale aux sociétés minières, ainsi qu'aux banques qui les finançaient, encourageaient l'exploration, d'où l'Australie tira de substantielles recettes. Dans la première moitié de la décennie 1920, l'approvisionnement extérieur de l'Australie en cuivre se faisait surtout depuis la mine de Laloki, près de Port Moresby. Après 1921 (fin de l'administration militaire), puis 1923 (entrée en vigueur du Mining Act), l'ouverture du Mandated Territory provoqua l'essor des explorations dans la région anciennement administrée par l'Allemagne, notamment dans la province de Morobe (Bassin de Wau), mais aussi, à plus petite échelle, à Koranga Creek et Edie Creek. En 1927, la New Guinea Goldfields, active à Edie Creek, puis à Golden Ridges, était introduite en bourse et levait un montant de 


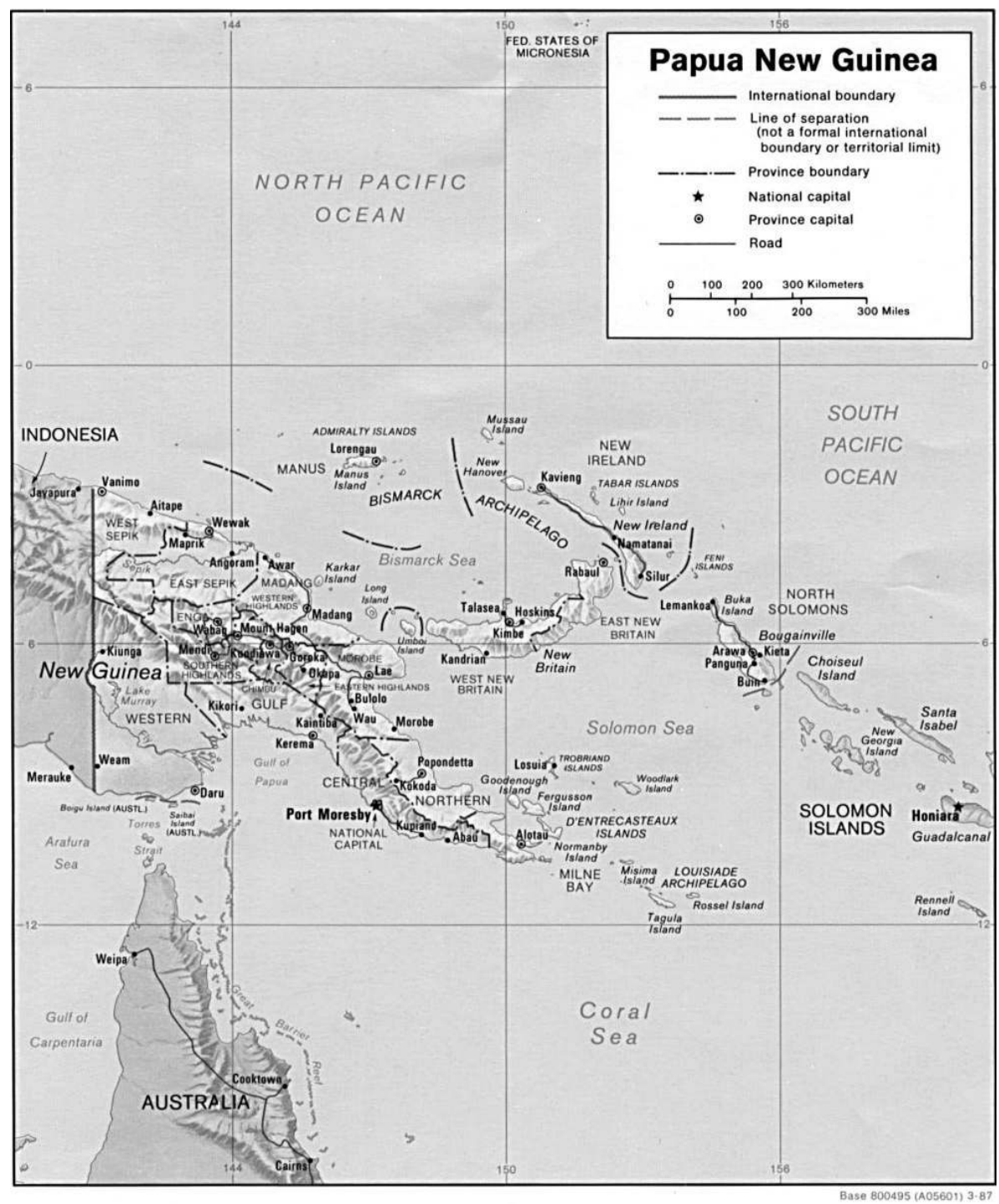

CARTE 1. - La Papouasie Nouvelle-Guinée (source : U.S. Central Intelligence Agency)

capitaux record à l'époque sur l'Australian Stock Exchange. De l'or était découvert à Bougainville en 1924 ; en 1938 à Porgera dans les Highlands... En valeur, l'or devenait désormais, avec le coprah, l'un des principaux postes d'exportation. Outre la New Guinea Goldfields Ltd, un autre protagoniste de la poussée australienne était la Colonial Sugar Refining de Sydney, liée à la Bank of New South Wales et qui avait pris, dès 1900, le contrôle de l'industrie sucrière en Australie.

$\mathrm{Au}$ cours de la crise des années 1930, qui poussa nombre de colons à la faillite, de grandes sociétés de négoce du sud de l'Australie arrivèrent en force, en rachetant des plantations en difficulté. À la même époque, les découvertes d'or avaient aussi attiré de gros investisseurs australiens, comme la BNG Mining Co. ou la Woodlark Island Gold Mining Co. Les intérêts économiques privés avançaient dans le cadre politique colonial, à l'image de ceux du groupe australien Burns, Philp \& Co., en position de quasi monopole dans les secteurs des transports et de l'alimentation notamment. En 1942, le nord de la Nouvelle-Guinée était envahi par les troupes japonaises dans le but de traverser l'île et de lancer l'attaque contre l'Australie ; mais l'opération échoua. Dès 1943, la contre-offensive états-unienne fit de la Nouvelle-Guinée la base arrière de la reconquête. Au sortir de la Seconde Guerre mondiale, l'onU reconduisait la tutelle australienne sur toute la partie orientale et l'archipel Bismarck. Papouasie et NouvelleGuinée étaient ainsi réunifiées par le PNG Act de 1949. C'est ce territoire qui accéda en 1973 à l'autonomie interne (self-governing) au sein de la fédération australienne, et finalement, deux ans 
plus tard, à l'indépendance, pour prendre le nom officiel de Papua Ningini. Entre-temps, la Papouasie Nouvelle-Guinée avait été placée dans la dépendance du marché australien (Hudson, 1971), qui ne s'ouvrait aux produits importés qu'au rythme des besoins de ses industries. C'est dans la métropole qu'étaient réinvestis les profits - pas à la périphérie. Cette spécialisation exclusive de la Papouasie Nouvelle-Guinée dans la production de biens primaires allait être si poussée qu'elle y empêcha tout processus d'industrialisation, y compris dans les secteurs dérivés de la culture de noix de coco (Antheaume et al., 1995). Ces rapports de domination économique furent garantis par un quadrillage militaire du pays, placé sous administration coloniale, assurant la « paix civile» et prenant appui sur des relais locaux, dont certaines missions religieuses.

Jusqu'au début de la décennie 1960, les structures productives et commerciales de la colonie restaient dominées par le coprah (et ses dérivés), le café et le cacao, cultures importées par l'industrie agro-alimentaire australienne. La phase d'exploitation moderne des gisements de Papouasie Nouvelle-Guinée commença dans la seconde moitié des années 1960, dans le sillage de cycles miniers australiens, et avec le soutien de l'État colonial par le biais de l'Australian Bureau of Mineral Resources. De nouveaux investisseurs firent leur apparition, comme Conzinc Rio Tinto of Australia, alors que d'importantes découvertes se succédaient : Panguna sur l'île de Bougainville (en 1964) par la Bougainville Copper Ldt, filiale australienne de Conzinc Rio Tinto ; Ok Tedi dans les Star Mountains (en 1968) par la Kennecott Cooper Corp., dépendant aussi du groupe Rio Tinto... Cette transnationale étendit ses prospections à Porgera au milieu des années 1960, rejointe par ses compatriotes Mount Isa Mines et New Guinea Goldfields. Encore sous lois coloniales, l'économie papouane-néo-guinéenne en fut bouleversée : dès 1975 , l'or et le cuivre devenaient les premiers postes d'exportation du pays. Cette même année, l'exercice de la Bougainville Copper Ldt exhibait les profits les plus élevés jamais réalisés par une société australienne (145 millions de dollars australiens de l'époque). L'essor des secteurs miniers, soutenu par Canberra et par les organisations internationales, accentua la spécialisation primaire du pays et aggrava sa dépendance vis-à-vis de l'Australie, dont le capital contrôla l'essentiel des exportations. L'économie de la colonie, désarticulée, limitant son rôle à servir de réservoir de main-d'œuvre, fournisseur de matières premières et zone de plantations, s'imbriquait progressivement dans les puissantes structures productive et commerciale de la métropole australienne, pour en devenir une sorte d'extension territoriale outre-mer. C'est plus de la moitié des investissements directs australiens qui y était destinée. Ainsi, à la veille de l'indépendance, prises dans leur ensemble, les entreprises australiennes et les filiales de firmes transnationales états-uniennes et britanniques implantées en Australie (Conzinc Rio Tinto of Australia, BHP, Carpenter, Burns Philp, Westpac, National Australia Bank, Comalco, Thiess, Boral, Dunlop, Pioneer, Rothmans...), auraient tiré de la Papouasie Nouvelle-Guinée des profits frôlant le quart du produit intérieur brut (PIB) de ce pays.

La base légale sur laquelle reposait l'économie coloniale de plantations et de mines s'appuya sur un ensemble de lois foncières et minières, qui ne furent pas sans conséquence pour les populations indigènes, l'accès aux terres et leur appropriation devenant un enjeu fondamental. L'enregistrement de terres coutumières et la tenue de cadastres de propriétés foncières ont donc constitué l'une des priorités absolues de l'administration coloniale australienne (Dale, 1976; Crocombe, 1987 ; Williamson, 1997). La pression exercée sur les communautés de l'intérieur provoqua de très fréquents affrontements avec les autochtones. Les sociétés australiennes de plantation et de négoce entrèrent dans un premier temps dans les plaines côtières et sur les îles, puis dans les Highlands où s'ouvrait l'opportunité de nouvelles conquêtes. Les parcelles de terre étaient réquisitionnées et louées à des colons, recrutant de la main-d'œuvre indigène, notamment pour la culture du café, qui gagnait du terrain - et avec elle la monétisation. Toutefois, la pénétration du territoire la plus vigoureuse fut liée, à partir des années 1950, aux prospections minières, soutenues par Canberra comme par les organisations internationales. En 1952, la Native Land Commission fut chargée d'enregistrer les terres coutumières, tandis que le Land Act de 1962 imposa un contrôle des transactions foncières, qui prohibait la vente ou la location de terres par des indigènes à titre individuel en dehors du droit coutumier et réservait ces opérations à l'État colonial. Les effets limités de ces enregistrements systématiques appelèrent des assouplissements. Le Land Tenure Conversion Act de 1963 visa à inciter les landowners à faire eux-mêmes enregistrer leurs parcelles. Mais à nouveau, les résultats furent peu probants et les résistances de communautés indigènes obstinées. Les autorités coloniales, soucieuses de sécuriser leurs titres de propriété sur des terres déjà acquises afin de satisfaire les demandes des colons planteurs (World Bank, 1978), lancèrent une nouvelle 
offensive contre la propriété collective en 1969, avec l'adoption des Land Titles Bills, puis des Land Reform Bills en 1971. En renforçant son contrôle du régime coutumier et en réintégrant les procédures d'enregistrement dans ses prérogatives exclusives, l'administration australienne revenait au système foncier le plus approprié à ses intérêts, au moment où la spécialisation papouane-néo-guinéenne se transformait en profondeur, passant de l'agriculture aux secteurs miniers et pétrolier. Les réactions de protestation obligèrent toutefois l'Australie à suspendre la " réforme » quelques mois avant l'accession du territoire au statut de self-governing. Repoussée sine die, l'application des lois foncières dut prendre d'autres formes; d'autant que le nouveau gouvernement (non encore indépendant) exprimait le souhait de récupérer les terres aliénées et de reconnaître constitutionnellement la propriété foncière coutumière (Bruce, 1988).

\section{La dépendance sous l'indépendance}

Les élections de 1972 amenèrent au pouvoir une coalition emmenée par le Papua New Guinea Union Party (Pangu Pati) de Michael Thomas Somare, partisan de l'indépendance. Les tensions s'exacerbèrent, tant au sein de l'alliance qu'avec l'Australie. Les enjeux étaient d'une telle ampleur, et les profits miniers et pétroliers si énormes, que l'Australie prépara avec soin l'indépendance de sa colonie. D'autres territoires de la région s'étaient déjà libérés à l'époque (Samoa en 1962, îles Cook en 1965, Nauru en 1968, Fidji en 1970, Niue en 1974...); mais l'importance stratégique de la Papouasie Nouvelle-Guinée pour les États-Unis et leurs alliés australiens était accentuée, dans le contexte complexe de la guerre froide, par la défaite au Viêt Nam. Le mouvement indépendantiste papouan-néo-guinéen s'était renforcé dans les années 1960, marquant une volonté de rompre avec le système colonial et, de la part des élites, de promouvoir des productions locales et une redistribution des profits qui leur soit plus favorable. Au lendemain de l'indépendance, qui intervint le 16 septembre 1975, l'une des priorités du gouvernement de M.T. Somare fut la mise en place d'un plan de développement, conduisant, notamment grâce à un contrôle étatique des secteurs clés, à la reprise en mains de l'économie par les autochtones (Denoon, 2005). Ce programme ambitieux, visant à renforcer la souveraineté économique du pays, n'atteignit pas ses objectifs, en dépit d'avancées relatives. L'échec était lié aux faiblesses de l'État central et du sentiment d'identité nationale, mais surtout à la stratégie appliquée. Car, au-delà des proclamations de principe, les mesures adoptées ne remirent pas en question la prépondérance des productions primaires d'exportation héritées de la colonisation et replacèrent au sommet des priorités l'attractivité d'un environnement institutionnel pour les investissements étrangers dans les secteurs miniers, très rentables : pression pour " réformer" le régime foncier et imposer la propriété privée individuelle, garantie de nonexpropriation, suppression de droits de douane, liberté de rapatriement des profits, privilèges fiscaux variés, construction d'infrastructures d'appui... La « sécurisation » du capital étranger, encouragée par la Banque mondiale (alors présidée par R. McNamara, ancien Secrétaire d'État des États-Unis de 1961 à 1968, en pleine guerre du Viêt Nam), fut garantie par une politique de stabilisation politique du territoire - et par la répression sociale. Les grèves de mineurs de 1975 à Panguna se soldèrent par des arrestations massives, tandis que le gouvernement central refusait à Bougainville de donner satisfaction aux revendications de groupes communautaires de landowners désireux de renégocier les termes du contrat passé avec la Conzinc Rio Tinto. Cette même année, la Papouasie Nouvelle-Guinée adhérait au Fonds monétaire international (FMI), à la Banque mondiale et à l'Accord général sur les Tarifs douaniers et le Commerce (GATT). La pérennisation de l'aide publique australienne assurait la continuité de relations privilégiées avec l'ancienne métropole.

Déterminés dans une large mesure par le contexte international, les cycles miniers rythmaient la vie économique du pays. À la fin de la décennie 1970, l'exploitation se concentrait surtout sur les gisements de Panguna et Wau, alors respectivement contrôlés par la Bougainville Copper Ldt et la New Guinea Goldfields. La brusque envolée des cours des métaux précieux du début des années 1980 relança les prospections (Neale, 2005). En 1988, Panguna était la plus grande mine de cuivre à ciel ouvert du monde, et l'une des plus importantes pour l'or. L'État papouan-néo-guinéen, actionnaire minoritaire, en tirait alors près du cinquième de ses recettes budgétaires. La mine dut cependant cesser de fonctionner en mai 1989 à la suite de l'éclatement d'un grave conflit avec les populations de Bougainville - très proches de celles des îles Salomon, mais détachées de ces dernières par la colonisation - ; conflit qui bascula dans la guerre civile durant près d'une décennie (Watts, 2007). Le relais pour l'or allait être pris, à partir 
de 1990, par les mines d'Ok Tedi, de Porgera et de Lihir, vers lesquelles étaient dirigés $80 \%$ des investissements étrangers, dopés par de généreux avantages fiscaux (Bank of Papua New Guinea, 1996). Le dynamisme des exportations d'or (et d'autres minerais stratégiques, tels l'argent, le cuivre, le nickel...) puis de pétrole, n'avait plus rien de commun avec l'évolution de celles de café ou d'huile de palme. Depuis la décennie 1980, événements internes (fermeture de Panguna, alourdissement de la dette publique) et chocs exogènes (surcoût de l'énergie importée, cours des matières premières exportées en baisse) combinèrent leurs effets pour aggraver la situation financière du pays. Les comptes interne et externe se dégradèrent par le creusement simultané des déficits du budget de l'État et des comptes courants, tandis que les réserves de change fondaient. Ces difficultés obligèrent encore le gouvernement à s'endetter auprès des bailleurs de fonds internationaux. Au final, la tentative de développement autocentré fut de si courte durée que le pays paraît être passé directement du colonialisme au néolibéralisme (Herrera et Tetoe, 2004a, 2010).

Les plans d'ajustement structurel (PAS) successivement appliqués en 1989, 1995 et 1999 marquent l'entrée de la Papouasie NouvelleGuinée dans l'ère néolibérale - soit la phase actuelle du capitalisme, caractérisée par une domination de la haute finance (Herrera, 2010). Premier ministre de 1988 à 1992, Rabbie Langanai Namaliu, nouveau leader du Pangu Pati, lança un premier PAS, camouflé en « réformes de restructuration » de l'économie : privatisation des entreprises publiques nationales, libéralisation du commerce extérieur, dérégulation des investissements, réorientation de la politique fiscale aux profit des entreprises (exonérations de taxes sur les importations pour les firmes, introduction de la TVA pour les ménages), flexibilisation du marché du travail... (Bank of Papua New Guinea, 1995). L'objectif était de rétablir la stabilité des indicateurs macroscopiques; laquelle fut obtenue au prix d'une contraction des dépenses publiques, d'une politique monétaire restrictive anti-inflationniste et d'une dévaluation de la monnaie (kina). Les résultats furent mitigés : si le taux de croissance se redressa sensiblement et les déséquilibres de la balance des paiements furent un moment contenus (Booth, 1995), les contraintes que cette stratégie imposait étaient asphyxiantes. Les causes structurelles de la dépendance n'étaient pas éradiquées. L'illusion ne pouvait durer : dès 1994, la détérioration des finances publiques obligea à se tourner de nouveau vers le FMI pour emprunter. La contrepartie fut un deuxième PAS, engagé en 1995 par le gouvernement de Julius Chan. La réalité de ce " programme de réforme structurelle » était celle d'une accentuation de la soumission écono-mique du pays, par la poursuite du démantèlement des régulations de l'État sur les investissements étrangers, le commerce extérieur, les prix et le marché du travail. Les mesures prises étaient conformes aux dogmes néolibéraux : relance des privatisations, taux de change flottant, suppression de droits de douane, libéralisation des prix, baisse et gel des salaires, hausse des frais à la charge des ménages pour l'éducation et la santé... En 1996, le pays entrait à l'Organisation mondiale du Commerce (OMC) - après avoir adhéré en 1993 à l' Asia Pacific Economic Cooperation (APEC) et obtenu un statut d'observateur au sein de l'Association des Nations de l'Asie du Sud-Est (ASEAN). Drastiques et très impopulaires, ces décisions n'empêchèrent pas la situation économique de se détériorer. Dans un contexte de forts chocs exogènes (crise asiatique, baisse des prix à l'exportation, sécheresses...), le gouvernement du Premier ministre Mekere Morauta (1999-2002), puis celui dirigé par le leader historique M.T. Somare (Premier ministre de 2002 à 2010, et chef du National Alliance Party) adoptèrent un troisième PAs, dicté de concert par les institutions internationales (y compris la Banque asiatique de Développement) et l'Australie. Sous l'impératif de "stabilisation », on accélérait la libéralisation et renforçait l'attractivité du territoire économique national pour le capital étranger - dans une économie où prédominaient désormais les exportations de pétrole et d'or. L'une des réformes les plus décisives des années 2000 fut celle du secteur financier, qui consolida l'indépendance de la Banque centrale et transféra à des propriétaires privés plusieurs établissements de crédit, compagnies d'assurance et caisses de retraite, à participation étatique jusque-là majoritaire. Dans ce système « modernisé », dominé par les oligopoles financiers (et où apparaissaient les fonds de pension), les profits dégagés par l'accumulation de capital fictif, ici comme ailleurs, explosaient. La dépendance perdurait, dans un contexte différent, celui du néolibéralisme.

Ce qui singularise véritablement la stratégie néolibérale en Papouasie Nouvelle-Guinée, où près des trois quarts de la population restent attachés aux cultures vivrières et plus de $95 \%$ des terres relèvent encore à l'heure présente du droit coutumier, c'est la priorité sans cesse accordée à l'enregistrement des terres communautaires et à l'établissement de cadastres de propriété foncière. Ce projet est justifié par 
l'argument selon lequel la persistance de droits ancestraux sur les sol et sous-sol constituerait un frein au développement (World Bank, 1989, Economist Intelligence Unit, années variées). D'où l'insistance avec laquelle sont exigées par les investisseurs étrangers et par les organisations internationales, la privatisation de la terre et la mise en place d'institutions garantissant des titres de propriété individualisés et soumis au droit capitaliste moderne. Le rapport de cette société à la terre et son attachement à la propriété collective se traduisent dans le fait que le droit foncier dérive de deux sources, constitutionnellement reconnues : le système de tenure capitaliste occidental, introduit par l'administration coloniale et aujourd'hui géré par l'État moderne (World Bank, 1978), et le système coutumier. Par conséquent, deux formes d'organisations foncières coexistent ; l'une relevant des systèmes traditionnels et qui couvre près de $97 \%$ des sols, l'autre correspondant aux terres d'État ou privées. Ainsi, en pratique, les terres dites « aliénées » et administrées par l'État, réservées à l'exploitation de mines et d'hydrocarbures en particulier, représenteraient aujourd'hui moins de $3 \%$ de l'ensemble du territoire national (Lalau, 1991). On comprend donc les convoitises auxquelles les propriétés communautaires indigènes sont soumises de la part des transnationales pour qu'elles soient privatisées. Chaque PAS a ainsi intégré un volet de "réforme foncière » - c'est-à-dire de privatisation des sols - comme condition des prêts : Land Mobilisation Program en 1989, conçu par le FMI et la Banque mondiale, appuyé par l'agence d'aide australienne (AUSAID) et prolongé à travers l'Australian Contribution to the Land Mobilisation Project (1993); Customary Land and Registration Bill en 1995, suspendu face au très vif mécontentement populaire, puis réintroduit en 1996, par l'enregistrement des terres dans les provinces pilotes de l'East Sepik et de l'East New Britain (Iatau et Williamson, 1997; Larmour, 2003) ; nouvelles privatisations en 2000, complétées par une série de lois spécifiques, par secteurs d'activités (Mining Act, Oil and Gas Act...), facilitant l'appropriation privée de terres et l'exploitation de leurs ressources naturelles... Au milieu des années 1990, les profits tirés de Papouasie Nouvelle-Guinée par les transnationales australiennes s'élevaient à près de 400 millions de dollars, contre 245 réalisés en Asie, 53 en Indonésie, 24 aux Fidji et 5 aux Salomon (Herrera et Tetoe, 2004a, b).

Face aux pressions des investisseurs et bailleurs de fonds, la position de l'État papouannéo-guinéen demeure plutôt ambigüe. Les opérations foncières doivent théoriquement toutes être effectuées par son intermédiaire, puisqu'il est seul autorisé à acheter et à louer des terres communautaires (non aliénées). La persistance de droits d'usage que le capital étranger ne peut ignorer pose problème aux autorités, notamment lors de l'attribution des gisements aux transnationales et de la construction d'infrastructures de soutien, ce qui exige la concession de droits d'exploitation de ressources à des investisseurs privés et l'aliénation par l'État de terres coutumières (Anderson, 2006). On observe cependant que cette structure foncière particulière, dominée par les formes traditionnellement collectives d'organisation sociale, n'a pas empêché l'essor des secteurs exportateurs des mines, des hydrocarbures et de l'agrobusiness. Il est vrai qu'existent certaines transactions foncières opérant hors de la sphère d'intervention étatique. Mais on constate surtout que, si l'État reconnaît partout les droits coutumiers de propriété des groupes communautaires - même là où le sous-sol est riche -, il n'en préserve en pratique l'intégrité que là où ces terres n'intéressent guère les investisseurs privés. Ailleurs, il s'empare également des sols pour en céder l'usage - y compris au capital étranger -, avec ce qui leur est attaché : gisements pétroliers, mines, plantations, forêts... (Herrera et Tetoe, 2004a, 2011). Une série de lois furent promulguées, par secteur d'activité, parmi lesquelles le Mining Act (1992) et l'Oil and Gas Act (1998) revêtent une importance majeure. L'accès aux richesses naturelles et leur exploitation par les transnationales étrangères se réalisent donc avec le soutien d'un État qui sait articuler ces processus d'appropriation sur les structures ancestrales de propriété collective, sans pour autant instaurer de marché foncier « libre ». Quand un projet est lancé, par exemple dans le cadre d'une joint venture, la solution de dédommagement est en général le versement de compensations monétaires aux landholders par les transnationales. D'évidence, les comportements de " chacun pour soi » gagnent du terrain, amenant des tensions, voire l'éclatement de certains clans. Simultanément, les groupes reconnus comme landholders - ou même actionnaires ! - en ont retiré argent et prestige (Godelier, 1982). En dépit des pressions exercées dans le sens de la division et de l'individualisation de la propriété de la terre, les gouvernements papouans-néo-guinéens qui se sont succédé ne sont pas parvenus à (ou n'ont pas vraiment souhaité) remettre en cause la propriété coutumière en raison de l'ampleur des soulèvements populaires qui les firent chaque fois reculer - jusqu'à l'heure présente. 


\begin{tabular}{|c|c|c|c|}
\hline $\begin{array}{l}\text { Nom de la mine et } \\
\text { localisation }\end{array}$ & $\begin{array}{l}\text { Minerais } \\
\text { extraits }\end{array}$ & Propriétairc(s) et nationalité(s) & $\begin{array}{l}\text { Partenaires locaux de joint } \\
\text { venture }\end{array}$ \\
\hline $\begin{array}{l}\text { Ok Tedi } \\
\text { (Western Province) }\end{array}$ & $\begin{array}{l}\text { or } \\
\text { cuivre } \\
\text { argent }\end{array}$ & $\begin{array}{l}\text { Jusqu'en } 2001 \text { : BHP-Billiton (Australie et } \\
\text { Royaume-Uni), lé compagnie minière } \\
\text { mondiale, propriétaire à hauteur de } 52 \% \text { et } \\
\text { Inmer Mining Corp. of Canada (Canada), } \\
\text { propriétaire à } 18 \% \\
\text { Općratcur depuis } 2002: \text { OTML }\end{array}$ & $\begin{array}{l}\text { Jusqu'en } 2001 \text { : propriété } \\
\text { de l'État à } 30 \% \text {, dont } 2.5 \% \\
\text { pour les landowners } \\
\text { Depuis } 2002 \text { : OTML (OK } \\
\text { Tedi Mining Led), détenu } \\
\text { par lc PNG Sustainable } \\
\text { Development Program Led }\end{array}$ \\
\hline $\begin{array}{l}\text { Lihir } \\
\text { (Lihir Island) }\end{array}$ & $\begin{array}{l}\text { or } \\
\text { cuivre } \\
\text { argent }\end{array}$ & $\begin{array}{l}\text { Newcrest Mining Ltd (Australie), } \\
\text { propriétaire à hauteur de } 100 \% \text { depuis } 2010 \\
\text { et le rachat de l'opérateur Lihir Gold Ltd. } \\
\text { (Rio Tinto [Royaume-Uni et Australie]) }\end{array}$ & $\begin{array}{l}\text { Mineral Ressources Lihir } \\
\text { (investisseurs privés et } \\
\text { institutionnels, groupes } \\
\text { communautaires) } \\
\end{array}$ \\
\hline $\begin{array}{l}\text { Hidden Valley - } \\
\text { Hamata } \\
\text { (Morobe Province) }\end{array}$ & $\begin{array}{l}\text { or } \\
\text { argent }\end{array}$ & $\begin{array}{l}\text { Morobe Goldfields, filiale de Harmony Gold } \\
\text { Mining Corp. (Africue du Sud) et Newcrest } \\
\text { Mining Ltd (Australie) }\end{array}$ & $\begin{array}{l}\text { Joint-venture à } 50-50 \\
\text { entre les deux compagnies }\end{array}$ \\
\hline $\begin{array}{l}\text { Sinivit } \\
\text { (New Britain) }\end{array}$ & $\begin{array}{l}\text { or } \\
\text { argent }\end{array}$ & $\begin{array}{l}\text { New Guinea Gold Corp. (Australie), } \\
\text { propriétaire à hauteur de } 90 \% \text {. Société mère : } \\
\text { Alcyone Resources Lud (ex-Macmim Silver) }\end{array}$ & $\begin{array}{l}10 \% \text { pour le Goldmines of } \\
\text { Niugini Holdings (détenu à } \\
\text { hauteur de } 22 \% \text { par le } \\
\text { partenaite NGG) }\end{array}$ \\
\hline $\begin{array}{l}\text { Simberi Island } \\
\text { (New Ireland) }\end{array}$ & or & $\begin{array}{l}\text { Allied Gold Lod (Australie), } \\
\text { propriétraire à } 100 \%\end{array}$ & \\
\hline $\begin{array}{l}\text { Kainantu } \\
\text { (Eastern Higblands) }\end{array}$ & or & $\begin{array}{l}\text { Highlands Pacific (Australie), } \\
\text { propriétaire à } 100 \%\end{array}$ & \\
\hline $\begin{array}{l}\text { Crater Mountain } \\
\text { (Eastern Highlands) }\end{array}$ & or & $\begin{array}{l}\text { Gold Anomaly Ltd (Australic) } \\
\text { propriétaire à } 90 \% \\
\text { et NGG Corp. (Australie) à } 10 \%\end{array}$ & \\
\hline $\begin{array}{l}\text { Simuku } \\
\text { (Madang Province) }\end{array}$ & cuivre & $\begin{array}{l}\text { Coppermoly Lud (Australie) } \\
\text { propriétaire à } 100 \%\end{array}$ & $\begin{array}{l}\text { Associée à NGG }(15 \%) \text { et } \\
\text { détenue à } 72 \% \text { par Barrick }\end{array}$ \\
\hline $\begin{array}{l}\text { Nakru } \\
\text { (Madang Province) }\end{array}$ & $\begin{array}{l}\text { cuivre } \\
\text { zinc }\end{array}$ & $\begin{array}{l}\text { Coppermoly Ltd (Australie) } \\
\text { propriétaire à } 100 \%\end{array}$ & \\
\hline $\begin{array}{l}\text { Yandera } \\
\text { (Madang Province) }\end{array}$ & $\begin{array}{l}\text { or } \\
\text { cuivre }\end{array}$ & $\begin{array}{l}\text { Marengo Mining Lrd (Australie) } \\
\text { propriétaire à } 100 \%\end{array}$ & \\
\hline $\begin{array}{l}\text { Woodlark } \\
\text { (Woodlark Island) }\end{array}$ & or & $\begin{array}{l}\text { Marengo Mining Lrd (Australic) } \\
\text { propriétaire à } 100 \%\end{array}$ & \\
\hline $\begin{array}{l}\text { Wafi-Golpu } \\
\text { (Morobe Province) }\end{array}$ & $\begin{array}{l}\text { or } \\
\text { cuivrc }\end{array}$ & $\begin{array}{l}\text { Harmony Gold (Afrique du Sud) } \\
\text { ct Newcrest Mining (Australic) }\end{array}$ & \\
\hline
\end{tabular}

TABleau 1. - Exemples de consortiums australiens exploitant des mines en Papouasie Nouvelle-Guinée en 2010

\begin{tabular}{|c|c|c|c|}
\hline $\begin{array}{l}\text { Nom du gisement et } \\
\text { localisation }\end{array}$ & $\begin{array}{l}\text { Hydro- } \\
\text { carbure }\end{array}$ & Principaux propriétaires et nationalité(s) & $\begin{array}{l}\text { Opérateur du } \\
\text { consortium et } \\
\text { nationalité }\end{array}$ \\
\hline $\begin{array}{l}\text { Kutubu } \\
\text { (Southern Highlands) }\end{array}$ & Pétrole & $\begin{array}{l}\text { Oil Search Ltd (à 71,95\%), } \\
\text { ExxonMobil }(14,52 \%) \text {, } \\
\text { Petroleum Resources Kutubu (6,75\%), } \\
\text { et Merlin Petroleum Co. }(6,78 \%)\end{array}$ & $\begin{array}{l}\text { Oil Search Ltd. } \\
\text { (PNG et Australie) }\end{array}$ \\
\hline $\begin{array}{l}\text { Gobe } \\
\text { Petroleum } \\
\text { Development } \\
\text { Field PDL-3 } \\
\text { (Southern Highlands) }\end{array}$ & Pétrole & $\begin{array}{l}\text { Southern Highlands Petroleum Ltd } \\
\text { (propriétaire à hauteur de 40,15\%), } \\
\text { Oil Search Ltd (à 36,36\%), } \\
\text { Santos }(15,92 \%) \\
\text { Cue PNG Oil Co Ltd }(5,57 \%) \\
\text { et Petroleum Resources Gobe }(2,0 \%)\end{array}$ & $\begin{array}{l}\text { Santos } \\
\text { South Australia Northern } \\
\text { Territory Oil Search } \\
\text { (Australie) }\end{array}$ \\
\hline $\begin{array}{l}\text { Moran } \\
\text { PDI-5 (Southern } \\
\text { Highlands) }\end{array}$ & Pétrole & $\begin{array}{l}\text { Oil Search (propriétaire à } 40,69 \%) \\
\text { Exxon Mobil Corp. }(36,81 \%) \\
\text { Eda Oil }(20,5 \%) \text { et landowners }(2 \%)\end{array}$ & $\begin{array}{l}\text { Exxon Mobil Corp. } \\
\text { par Esso Australia Ltd }\end{array}$ \\
\hline $\begin{array}{l}\text { Hides } \\
\text { (Southern Highlands) }\end{array}$ & Gaz & $\begin{array}{l}\text { Exxon Mobil Corp. }(41,6 \%) \text {, } \\
\text { Oil Search Ltd }(34,1 \%), \\
\text { Santos }(17,7 \%) \text {, Nippon Oil }(1,8 \%) \text {, } \\
\text { MRDC et landowners }(1,2 \%)\end{array}$ & $\begin{array}{l}\text { Exxon Mobil Corp. } \\
\text { par Esso Highlands Ltd } \\
\text { et Esso Australia Ltd }\end{array}$ \\
\hline
\end{tabular}

TABLeAu 2. - Exemples de consortiums australiens exploitant du pétrole et du gaz en Papouasie Nouvelle-Guinée en 2010

\section{Les mécanismes de la dépendance économique}

Les trois quarts de la force de travail papouane-néo-guinéenne se consacrent aujourd'hui encore aux cultures de subsistance, dont les produits sont autoconsommés ou vendus sur des marchés locaux - et très marginalement exportés. Mais ce sont les secteurs miniers et énergétiques, fortement capitalistiques, comptant pour près de $20 \%$ du PIB (Herrera et Tetoe, 2010), qui constituent les moteurs de croissance de la Papouasie Nouvelle-Guinée. À la fin de la décennie 2000, la production d'or dépassait 60 tonnes par an, soit le $11^{\mathrm{e}}$ rang mondial (www. mineral.gov.png). Le pays disposerait des troisièmes réserves aurifères de la planète. Il est également un gros producteur de cuivre, occupant le $13^{\mathrm{e}}$ rang mondial (avec 200000 tonnes par an). La majeure partie de cet or est raffinée à Perth, puis réexportée à partir de l'Australie (Department of Foreign Affairs and Trade, 2011). Les principaux systèmes miniers en activité se concentrent dans la cordillère des Highlands et les îles environnantes: Ok Tedi (Western Province), Lihir (Nouvelle-Bretagne), Porgera (Enga), plus l'ensemble de Morobe (Department of Mining, 2010). Comme le montre le tableau 1, l'Australie est omniprésente dans la structure de propriété du capital des consortiums miniers: le site d'Ok Tedi, d'où sont extraites près de 500000 onces d'or (soit 15,5 tonnes) était jusqu'en 2001 majoritairement détenu par le premier groupe minier mondial, 
l'australo-britannique BHP-Billiton - qui se retira après un scandale écologique -; celui de Lihir, qui produit à l'heure actuelle près de 900000 onces d'or (près de 28 tonnes), appartient depuis 2010 à Newcrest Mining Ltd., cinquième groupe mondial producteur d'or, domicilié à Melbourne; ce même groupe exploite en joint venture Wafi-Golpu (or et cuivre) et Hidden Valley - Hamata (or et argent) dans la Morobe Province ; l'australienne Coppermoly détient les mines de Simuku (cuivre) et Nakru (cuivre et zinc) dans la Madang Province, et sa compatriote Marengo celle de Yandera (or et cuivre) ; la Gold Anomaly contrôle le gros de l'extraction à Crater Mountains (or), et Highlands Pacific toute celle de Kainantu (or) dans les Eastern Highlands; New Guinea Gold est propriétaire des gisements de Sinivit (or et argent) en Nouvelle Bretagne, Allied Gold de ceux de Simberi (or) en Nouvelle Irlande, Gold Anomaly de Crater Mountain dans les Eastern Highlands - trois firmes australiennes. Il en va de même pour le contrôle des consortiums pétroliers et gaziers opérant dans les Southern Highlands (tableau 2) : Oil Search à Kutubu, Santos à Gobe et la filiale australienne d'Esso à Moran pour le pétrole (www.petroleum.gov.png); Esso Australia Ltd, pour le compte de l'Exxon Mobil Corp., à Hides pour le gaz naturel. On retrouve ces entreprises dans le projet en cours du $P N G$ Liquid Niugini Gas (http://liquidnuiginigas. com/project), d'un montant estimé à 15 milliards de dollars états-uniens...

Dans ces conditions, l'économie papouanenéo-guinéenne reste extrêmement dépendante de l'extérieur, tout spécialement de l'Australie (Amarshi et alii., 1979). Le total de ses échanges extérieurs (somme des exportations et des importations) représente selon les années entre 55 et $75 \%$ de la valeur du PIB - contre $35 \%$ en Australie et $54 \%$ en Nouvelle-Zélande (World Bank, 2011). Son premier partenaire commercial est, de loin, l'ancienne tutelle coloniale, grâce notamment au cadre bilatéral libreéchangiste du Trade and Commercial Relations Agreement et à celui, plus large, du South Pacific and Regional Trade Agreement (SPARTECA). Ainsi, en 2010, près de la moitié des importations de marchandises (pour un montant de plus d'un milliard de dollars) provenait d'Australie, qui trouvait en Papouasie Nouvelle-Guinée un débouché assuré pour ses produits (tableau 3). L'importance du marché australien, proche, vaste, dynamique, est aussi perceptible à l'exportation : plus d'un quart des exportations y était destiné en 2010 - mais pour une gamme de biens beaucoup plus limitée. La dépendance s'observe dans la composition même des échanges. Au niveau agrégé, les premiers postes d'importations sont les combustibles, les biens d'équipement (intrants, machines, matériels de transport...) et les produits chimiques. Une grande partie de ces importations est commandée, non pas tant par la logique d'accumulation du capital domestique, mais plutôt par celle des investissements étrangers dans les secteurs minier et pétrolier - moteurs des exportations. Les importations de biens de consommation n'arrivent que bien après, traduisant le caractère non prioritaire de la satisfaction des besoins fondamentaux de la population, ainsi que certains comportements de démonstration (achat de produits de luxe) des couches les plus favorisées de la société (Gewertz et Errington, 1999) - la part dans la consommation des $10 \%$ de ménages disposant des plus hauts revenus étant estimé à plus de $40 \%$ (UNDP, 2008). La structure des exportations papouanes-néo-guinéennes est presque exclusivement composée de produits primaires - secteurs où les transnationales sont en position de force (tableau 4). Il s'agit, pour plus des deux tiers, de produits minéraux non transformés (or, pétrole brut, cuivre, argent essentiellement) et, pour un quart environ, de produits agricoles non élaborés (café, cacao, huiles de palme et de coprah, bois et matières forestières, produits de la mer...). Les échanges avec l'Australie sont symptomatiquement déformés par le poids des « transactions spéciales et articles confidentiels » (c'est-à-dire secret défense). Quant au commerce avec les États-Unis, ce sont les ventes de pétrole qui arrivent en tête à l'exportation, suivies par l'or; et, côté importations,

\begin{tabular}{|r|c|r|c|}
\hline $\begin{array}{r}\text { Partenaires à } \\
\text { l'exportation }\end{array}$ & $\begin{array}{c}\text { \% des } \\
\text { exportations }\end{array}$ & $\begin{array}{r}\text { Partenaires à } \\
\text { l'importation }\end{array}$ & $\begin{array}{c}\% \text { des } \\
\text { importations }\end{array}$ \\
\hline Australie & 27,9 & Australie & 42,1 \\
\hline Japon & 9,1 & Singapour & 13,1 \\
\hline Chine & 7,1 & Chine & 7,9 \\
\hline Philippines & 3,0 & Japon & 6,6 \\
\hline
\end{tabular}

TABleau 3. - Place de l'Australie parmi les principaux partenaires commerciaux de la Papouasie Nouvelle-Guinée en 2010 (source : Department of Foreign Affairs and TradeGovernment of Australia, 2011)

\begin{tabular}{|l|c|c|l|}
\hline $\begin{array}{l}\text { Principaux produits } \\
\text { exportés }\end{array}$ & $\begin{array}{c}\text { Valeur des } \\
\text { exportations }\end{array}$ & $\begin{array}{c}\text { Valeur des } \\
\text { importations }\end{array}$ & Principaux produits importés \\
\hline 1.Or & 2015 & 452 & l. Pétrole \\
\hline 2. Pétrolc & 1179 & 106 & 2. Équipcments de génic civil \\
\hline 3. Argent et platine & 379 & 78 & 3. Machines spécialisées \\
\hline 4. Café et substituts & 55 & 69 & 4. Viande (hors bouf) \\
\hline
\end{tabular}

TABleau 4. - Composition des échanges commerciaux de la Papouasie Nouvelle-Guinée avec l'Australie en 2011 (en milliers de dollars australiens) (source: Department of Foreign Affairs and Trade-Government of Australia, 2012) 
les machines, biens d'équipement et matériels de transport. Depuis le début de la décennie 2000, les échanges commerciaux se sont aussi intensifiés avec la Chine, devenu le troisième partenaire de la Papouasie Nouvelle-Guinée, à l'importation et à l'exportation.

La forte dépendance de l'appareil productif papouan-néo-guinéen, inséré dans un système d'échanges régionaux polarisant ne répondant pas aux exigences de développement du pays, est encore visible - en raisonnant sur la moyenne des années 2000-2010 - dans la dissymétrie existant entre l'excédent de la balance commerciale et le déficit de la balance des invisibles (ou services échangés avec l'extérieur). Le premier est dû à l'essor de la demande extérieure ; le second aux paiements des dividendes sur investissements directs étrangers et des intérêts de la dette. Mais les profits sortant du pays creusent les déficits chroniques de la balance des comptes courants et de la balance financière. Ces transferts de surplus s'effectuent en grande partie au bénéfice de banques et de firmes australiennes (tableau 5). À titre d'exemple, l'exercice 2008 révèle que $55 \%$ des dividendes versés aux détenteurs étrangers d'actifs allaient à des propriétaires de capitaux australiens. En 2005, le montant des dividendes prélevés par des entités privées australiennes dépassait 500 millions de kina (soit plus de 160 millions de dollars états-uniens) ; pour l'exercice précédent, il s'élevait à 490 millions de kina (dont 219 millions revenant à des propriétaires états-uniens, devant ceux de nationalité britannique). Quant à la répartition sectorielle des dividendes, $75 \%$ d'entre eux provenaient, sans surprise, des mines et du pétrole - à comparer à $9 \%$ pour l'industrie et $5 \%$ pour l'agriculture (Bank of Papua New Guinea, 2010). L'équilibre de la balance des paiements est donc essentiellement réalisé grâce aux flux entrants de l'investissement direct étranger et de l'aide publique extérieure. Entre 1990 et 2000 , les investissements directs à destination de la Papouasie Nouvelle-Guinée ont fortement fluctué, mais sont restés largement positifs. Au début de la décennie 2000, leur part dans la formation brute de capital fixe approchait $50 \%$. Ils étaient en majorité australiens (suivis par ceux des États-Unis et du Royaume-Uni), et principalement investis dans les mines et le pétrole. L'aide publique provient, de façon privilégiée, de l'Australie - secondairement du Japon. La logique de ces flux privés et publics paraît claire: l'État papouan-néo-guinéen assure l'ouverture des marchés locaux et la privatisation d'entreprises qu'il détenait au profit du capital privé, surtout étranger ; ce dernier entre dans les structures de propriété du capital du pays et opère les transferts de surplus de l'économie locale vers les firmes et banques étrangères ; la coopération australienne fournit à cet État l'aide nécessaire au rééquilibrage des déficits publics et d'une balance des paiements déstructurée par ces transferts ves l'extérieur, tout en approfondissant la stratégie néolibérale - et en contenant les résistances locales. La Papouasie Nouvelle-Guinée jouit du statut de premier pays récipiendaire de l'aide publique australienne, avec 415 millions de dollars australiens reçus pour l'année 2010 via l'Australian Government's Overseas Aid Program et 457 millions supplémentaires au titre de l'Official Development Assistance - soit, au total, plus de la moitié de l'aide bilatérale accordée par Canberra (http:// www.ausaid.gov.au/anrep/rep10/program1_lpng. $\mathrm{html}$ ). Depuis 1975, ses montants cumulés dépasseraient les cinq milliards de dollars. L'adoption de réformes néolibérales, ainsi que le respect des principes de la « bonne gouvernance » - le pays étant régulièrement classé parmi les plus corrompus au monde (154 $\mathrm{rang}$ sur 182 selon le Corruption Perceptions Index de Transparency International en 2011), conditionnent l'aide bilatérale (Herrera, 2004). Par exemple, ce n'est qu'une fois renouvelé l'accord stand-by (c'est-àdire de confirmation de crédit) de la Papouasie Nouvelle-Guinée avec le FMI en mars 2000 que put être débloquée l'aide de l'Australie trois mois plus tard, permettant l'octroi de nouveaux prêts des organisations internationales en décembre de la même année.

Dans ce schéma, la dette extérieure joue un rôle clé. Cette dernière a beaucoup augmenté

\begin{tabular}{|l|r|r|r|r|}
\hline Pays & $\mathbf{1 9 9 5}$ & $\mathbf{2 0 0 1}$ & $\mathbf{2 0 0 8}$ & 2010 \\
\hline Australie & 1548 & 956 & 1027 & 2304 \\
\hline Royaume-Uni & 168 & 97 & 92 & 83 \\
\hline Bahamas & - & 108 & 84 & 76 \\
\hline Malaisie & - & 73 & 76 & 66 \\
\hline Singapour & - & 11,5 & 73 & 66 \\
\hline Japon & 35 & 62 & 765 & 689 \\
\hline Canada & 19 & 24 & 44 & 39 \\
\hline Chine (Hong Kong) & - & 26 & 31 & 28 \\
\hline Bermudes & - & 38 & 29 & 27 \\
\hline États-Unis & 84 & 65 & 21,3 & 19 \\
\hline Corée du Sud & - & - & 11 & 10 \\
\hline Autres & - & - & 333 & 567 \\
\hline Total (en valeur) & 306 & 1460 & 1152 & 3974 \\
\hline Total (en \% du PIB) & 36,6 & 27,2 & 22,4 & 38,3 \\
\hline
\end{tabular}

TABleau 5. - Portefeuilles d'actifs à propriété étrangère en Papouasie Nouvelle-Guinée selon le pays d'origine en 1995, 2001, 2008 et 2010 (en millions de dollars autraliens) (source : Bank of Papua New Guinea) 
durant les années 1990, consécutivement à l'alourdissement des besoins de financement liés à l'essor des activités minières (site de Lihir) et pétrolière (complexe de Kutubu); puis encore brutalement à la fin de la décennie, à la suite de la crise asiatique de 1997-1998 et de l'appel aux organisations internationales (Bank of Papua New Guinea, 2009). Après un pic atteint en 2003-2004, au-dessus de 20 milliards de kina, un repli a permis à cette dette de revenir en $2009 \mathrm{au}$ niveau de 1999. Néanmoins, les flux occasionnés par le service de la dette extérieure restent aujourd'hui supérieurs à ceux des aides publiques reçues. Dans les années 2000, le stock de dette publique, presque exclusivement de long terme, équivalait à peu près à la valeur du PIB, soit une fois et demie celle des exportations et trois fois le total du budget de l'État. Sur cette somme, les trois quarts étaient dus aux organisations internationales (à hauteur des deux tiers publiquement garantis) - le reste correspondant à des dettes publiques bilatérales contractées pour l'essentiel auprès de l'Australie, plus marginalement de banques privées. Ajoutés à ceci, les engagements sont aussi importants vis-à-vis $\mathrm{du}$ FMI. Pourtant, la dette domestique, dont le remboursement oblige à alourdir la dette extérieure, a tendance à s'accroître, en particulier sous forme de bons du Trésor, détenus par des investisseurs institutionnels étrangers, mais également par des propriétaires nationaux, au nombre desquels figurent de hauts responsables militaires. Dans un contexte où la fonction régalienne de défense nationale a été partiellement " déléguée » à l'Australie et à des sociétés militaires privées pour la protection des sites stratégiques pour le capital étranger (notamment sous le Premier ministre Julius Chan en 1997), le rôle de certaines fractions de l'armée papouane-néo-guinéenne a été décisif dans l'orientation néolibérale du cours de la politique économique (Herrera et Tetoe, 2010). En parallèle, force est de constater que l'aide militaire australienne (de l'ordre de $15 \%$ du total) occupe une place centrale dans le dispositif visant à convaincre les autorités papouanes-néoguinéennes d'adopter une ligne plus conforme aux intérêts stratégiques de Canberra - notamment en faveur du nucléaire et en matière d'environnement (pression pour refuser de signer le protocole de Kyoto). L'évolution politique de la Papouasie Nouvelle-Guinée demeure toujours difficile à prévoir et des tensions couvent dans certaines îles mélanésiennes, comme Bougainville. L'impact des contraintes extérieures et du degré élevé de dépendance a été déterminant sur la trajectoire prise par l'économie papouane- néo-guinéenne, marquée par la relative faiblesse et l'instabilité de sa croissance économique à long terme. C'est encore la dépendance vis-à-vis de l'extérieur qui éclaire les rigidités caractérisant les finances publiques. Côté recettes, les revenus générés par l'impôt sur les sociétés ont augmenté (jusqu'en 2008 et le déclenchement de la crise), mais le régime de taxation des dividendes n'en reste pas moins très accommodant pour les transnationales - encouragées par maintes incitations fiscales (exonérations partielles de taxes à l'importation de biens d'équipements, notamment). Du côté des dépenses, les fonds publics finançant les grands travaux de soutien du capital privé absorbent des parts du budget de l'État disproportionnées, tandis que les infrastructures vitales à l'agriculture vivrière ou au commerce domestique sont négligées. Même des projets conçus dans un esprit qui pourrait rappeller la stratégie d'industrialisation par substitution aux importations de jadis sont le plus souvent pensés comme devant servir l'expansion des investissements étrangers (par exemple, la production de ciment, soumise aux impératifs des secteurs d'exportation).

\section{Conclusion}

En conséquence, les besoins immédiats de la population sont sacrifiés et les indicateurs sociaux de la Papouasie Nouvelle-Guinée figurent parmi les plus bas du monde. Selon les données de l'unESCO, le taux d'alphabétisation (des plus de 15 ans) était de $57,8 \%$ en 2009, et $53,4 \%$ seulement pour les femmes ( $c f$. http: //hdrstats.undp.org/en/indicators/93.html). L'éducation est loin d'être universalisée : au début de la décennie 2000, moins de $40 \%$ des enfants étaient scolarisés (UNESCO, 2009). En matière de santé, les indicateurs sont aussi très pauvres. L'espérance de vie à la naissance n'y était en moyenne que de 60,7 ans en 2008, contre 68,7 aux Fidji, 70,5 en Indonésie et 81,4 en Australie (UNDP, 2008). Le taux de mortalité infantile frôlait les $80 \%$, contre $35 \%$ en Indonésie et $18 \%$ aux Fidji. Les taux moyens de vaccination restent inférieurs à ce qu'ils sont en Océanie. Les inégalités sociales sont fortes, avec un rapport entre les revenus des $10 \%$ de la population les plus pauvres et les $10 \%$ les plus riches de 1 à 22 et un degré de concentration des revenus - dit coefficient de mesure statistique des inégalités de Gini - parmi les plus élevés du monde, de l'ordre de 0,51 (World Bank, 2011), tandis que près de $70 \%$ de la population urbaine vivrait sous le seuil de pauvreté. 
On comprend dès lors à la fois l'impact destructeur que produit l'extension de rapports fonciers capitalistes sur la survie de liens ancestraux de solidarité et les motifs qui ont poussé ces dernières années de larges composantes de la société (travailleurs du privé, fonctionnaires, petit peuple des villes, étudiants, militants associatifs écologistes, féministes ou religieux, communautés de landowners, soldats même...) à se révolter contre la rigueur des PAs, la privatisation des ressources naturelles (l'eau et les forêts, qui intéressent aussi l'Australie) et les réformes du statut des terres (Herrera et Tetoe, 2012). L'ampleur de ces résistances pose la question de la recherche d'alternatives au néolibéralisme en forme de stratégie garantissant un développement équilibré, autocentré et bénéficiant à un peuple désireux d'être maître de son devenir collectif. La dépendance de la Papouasie Nouvelle-Guinée est certes extrême vis-à-vis des bailleurs de fonds internationaux, et la crise financière actuelle a récemment dégradé la structure des échanges du pays, avec l'apparition d'un déficit de la balance commerciale en 2010. Les marges de manœuvre du gouvernement sont étroites, dans un pays dont le produit intérieur brut (de 14,9 milliards de dollars étatsuniens en 2010, en parité de pouvoir d'achat) (http://www.economywatch.com/economic-statistics/country/Papua-New-Guinea/) dépasse à peine la moitié du chiffre d'affaires (26 milliards de dollars) de Rio Tinto, l'une des firmes transnationales opérant sur son sol. Elles ne sont cependant pas inexistantes; d'autant que les recettes des exportations minières et pétrolières pourraient permettre d'envisager la redistribution de la rente dans le sens d'une meilleure satisfaction des besoins sociaux, en s'appuyant sur un régime foncier où les terres cultivables échappent encore largement au droit capitaliste moderne.

\section{BIBLIOGRAPHIE}

Amarshi Azeem, Kenneth Good et Rex Mortimer, 1979. Development and Dependency: the Political Economy of Papua New Guinea, Melbourne, Oxford University Press.

Antheaume Benoit, Joël Bonnemaison, Michel Bruneau et Christian Taillard, 1995. Asie du Sud Est-Océanie, Paris, Belin - Reclus.

ANDERSON Tim, 2005. Challenging Integrated Conservation and Development in Papua New Guinea, Pacific Economic Bulletin 20, 1, pp. 56-66.

Australia Government, 2009. Defending Australia in the Asia Pacific Century: Force 2030, Department of Defence (disponible sur: www.defence.gov.au).
Bank of Papua New Guinea, années variées. Quaterly Economic Bulletin, Port Moresby (disponible sur www.bpng.gov.pg).

Bоoтн Anne, 1995. Development Challenges in a Poor Pacific Economy: The case of Papua New Guinea, Pacific Affairs 68, 2, pp. 207-230.

BRUCE John W., 1988. A Perspective on Indigenous Land Tenure Systems and Land Concentration, Downs and Reyna, pp. 23-52.

Crocombe Ron, 1987. Land Tenure in the Pacific, University of the South Pacific, Suva.

Dale Peter F., 1976. Cadastral Surveys within the Commonwealth, London, Her Majesty's Stationery Office, Overseas Research Publication 23.

Department of Foreign Affairs and Trade, 2011. Australia 2010: Composition of Trade.

DePARTMENT OF Mining, années variées. Information Booklets, Port Moresby (disponible sur www.mineral.gov.png).

Denoon, Donald, 2005. A Trial Separation: Australia and the Decolonization of Papua New Guinea, Canberra, Pandanus Books.

ECONOMIST INTELLIGENCE UNIT, années variées. Country Profile, Londres (disponible sur www.eiu. com).

Gewertz Deborah B. et Frederick K. ERrington, 1999. Emerging Class in Papua New Guinea - The Telling of Difference, Cambridge, Cambridge University Press.

Gille Bernard et Pierre-Yves Toullelan, 1999. De la conquête à l'exode, Pirae, Tahiti, Au Vent des Îles.

GODELIER Maurice, 1982. La production des grands hommes. Pouvoir et domination masculine chez les Baruya de Nouvelle Guinée, Paris, Fayard.

Herrera Rémy, 2004. Good Governance Against Good Government?, Third World Economics 324, pp. 14-15.

—, 2007. Australie : l'impérialisme oublié, Afrique Asie 7/8, pp. 58-61.

_, 2010. Un Autre Capitalisme n'est pas possible, Paris, Syllepse.

Herrera Rémy et Poeura Tetoe, 2004a (septembre). Land Property and Development: a Comparative Study of Papua (Indonesian Irian Jaya) and Papua New Guinea, papier présenté à la conférence de la European Association of Southeast Asian Studies (EURoseas), Paris.

—, 2004b (septembre). FDIs' Socio-economic Effects in Papua New Guinea, papier présenté à la conférence de l'Association française pour la Recherche sur l'Asie du Sud-Est (AFRASE), Paris.

_, 2010. L'économie papouane-néo-guinéenne entre dépendance et résistances, mimeo CNRS, UMR 8174 Centre d'Économie de la Sorbonne, Université de Paris 1 Panthéon-Sorbonne, Paris.

_, 2011. Le capital étranger dans le secteur des mines en Papouasie Nouvelle-Guinée, Économies et Sociétés (série F) 45, 2, pp. 321-360. 
—, 2012. Le paradoxe Papua Niugini : Archaïsme de la propriété de la terre, modernité des résistances paysannes?, Revue française de Socio-économie 9, pp. 133-152.

Hudson, William James, 1971. Australia and Papua new Guinea, Sydney, Sydney University Press, $198 \mathrm{p}$.

Iatau Marie T. et Ian P. Williamson, 1997. An Introduction to the Use of Case Study Methodology to Review Cadastral Reform in Papua New Guinea, Commission 7 Symposium, $64^{\text {th }}$ Permanent Committee of the International Federation of Surveyors, Singapour.

InSEe, 1951. L'Australie, Mémento économique, Paris, Presses universitaires de France.

LARMOUR Peter, 2003. Land Registration in Papua New Guinea: Competing Perspectives, discussion paper, State, Society and Governance in Melanesia, Canberra, Australian National University, Research School of Pacific and Asian Studies.

LEBLIC Isabelle, 1993. Les Kanak face au développement : la voie étroite, Grenoble, Presses universitaires de Grenoble avec le concours de Agence de développement de la culture Kanak, 420 p.

Maude, Henry Evans, 1981. Slavers in Paradise: the Peruvian Labour Trade in Polynesia (1862-1864), Stanford-Suva, Stanford University Press-Institute of Pacific Studies.
Molesworth Bevil Hugh, 1917. Kanaka Labour in Queensland (1863-1871), Journal of the Royal Historical Society of Queensland 1, 3, pp. 140-154.

NeALE Trevor, 2005. Historical Overview of Mining in PNG, Melanesian Resources (disponible sur : http://infomine.com/).

PANOFF Michel, 1979. Travailleurs, recruteurs et planteurs dans l'archipel Bismark de 1885 à 1914, Journal de la Société des Océanistes 64, pp. 159-173.

Pons Xavier, 1994. Le géant du Pacifique, Paris, Economica.

UndP, années variées. Human Development Report, Geneva.

Unesco, 2009. Global Age-specific Literacy Projections, Institute for Statistics, Paris.

Watts Max, 2007. Chronology of Bougainville Civil War, Mimeo, Sydney.

Williamson I. P., 1997. The Justification of Cadastral Systems in Developing Countries, Geomatica 51, 1, pp. 21-36.

World BANK, 1978. PNG: Economic Ssituation and Prospects for Developement, Washington D.C.

—, 1989. Land Mobilisation Project, Report 7592, Department of Lands, Port Moresby.

-, années variées. World Development Report, Washington D.C.

-, années variées. World Developement Indicators, Washington D.C. 